Received: 21 July 2017

Accepted: 20 October 2017

Published online: 06 November 2017

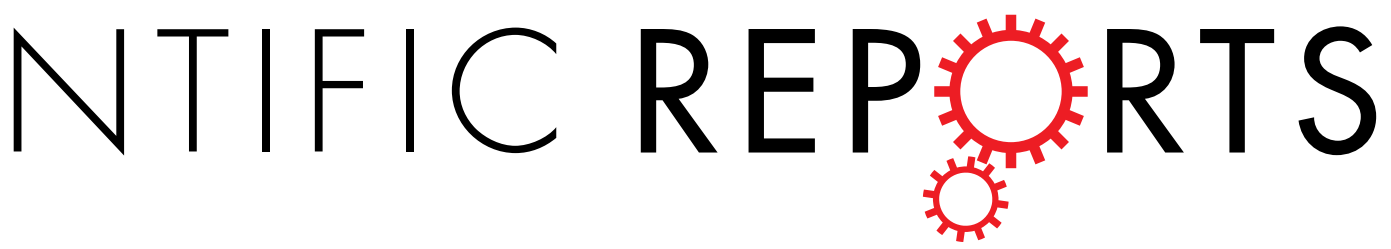

\title{
OPEN Identification of claudin-4 binder that attenuates tight junction barrier function by TR-FRET-based screening assay
}

Akihiro Watari ${ }^{1}$, Miki Kodaka ${ }^{1}$, Koji Matsuhisa ${ }^{2}$, Yuta Sakamoto $^{1}$, Kota Hisaie ${ }^{1}$, Norihito Kawashita ${ }^{3}$, Tatsuya Takagi ${ }^{1,4}{ }^{4}$, YoshiakiYamagishi ${ }^{5}$, Hidehiko Suzuki ${ }^{6}$, Hirofumi Tsujino ${ }^{1}$, Kiyohito Yagi ${ }^{1}$ \& Masuo Kondoh ${ }^{1}$

Claudins are key functional and structural components of tight junctions (TJs) in epithelial cell sheets. The C-terminal fragment of Clostridium perfringens enterotoxin (C-CPE) binds to claudin-4 and reversibly modulates intestinal $T J$ seals, thereby enhancing paracellular transport of solutes. However, the use of C-CPE as an absorption enhancer is limited by the molecule's immunogenicity and manufacturing cost. Here, we developed a high-throughput screening system based on the TimeResolved Fluorescence Resonance Energy Transfer (TR-FRET) method to identify claudin-4 binders in a library collection of 32,560 compounds. Thiostrepton, identified from the screen, decreased transepithelial electrical resistance and increased flux of 4-kDa fluorescein isothiocyanate-labelled dextran (FD-4) in Caco-2 cell monolayers, a model of intestinal epithelium. Thiostrepton changed the expression, but not the localisation, of TJ components. Treatment of rat jejunum with thiostrepton increased the absorption of FD-4 without tissue toxicity, indicating that thiostrepton is a novel claudin- 4 binder that enhances intestinal permeability. The screening system may therefore be a useful tool for identifying claudin- 4 binders to enhance drug absorption in mucosa.

Biopharmaceuticals, including peptides, proteins, antibodies and nucleic acids, play pivotal roles in the development of new drugs with breakthrough effects. The proportion of the drug market occupied by biopharmaceuticals is increasing yearly. Currently, biopharmaceuticals are administered primarily by injection, despite the pain and potential adverse effects (e.g., anaphylaxis) associated with this route. Therefore, the development of biopharmaceuticals that can be given through oral, nasal, pulmonary or percutaneous administration is required to avoid the risks of injection. However, biopharmaceuticals show remarkably low rates of absorption through mucosal or cutaneous epithelium owing to their hydrophilicity and high molecular weight ${ }^{1}$, and we need to develop a method for increasing their absorption to ensure sufficient bioavailability ${ }^{2}$.

Tight junctions (TJs), which are formed in the interstices between adjacent epithelial cells, seal the paracellular space to restrict the passage of solutes through the epithelial cell sheet ${ }^{3,4}$. Because TJs limit drug permeation through paracellular spaces, regulating TJ seals is an efficacious strategy for improving drug absorption ${ }^{5}$. A variety of substances controlling paracellular permeability have been developed ${ }^{5-12}$, and several of these bind claudin, a major component of the TJ barrier ${ }^{13,14}$. One of the most efficient is the C-terminal fragment of Clostridium perfringens enterotoxin (C-CPE), which binds to claudin- 4 and can reversibly weaken the TJ barrier ${ }^{15}$. In rats, C-CPE increases the jejunal absorption of 4 -kDa dextran (FD-4) by 400-fold over that of the currently used clinical absorption enhancer, sodium caprate ${ }^{8}$.

${ }^{1}$ Graduate School of Pharmaceutical Sciences, Osaka University, 1-6 Yamadaoka, Suita, Osaka, 565-0871, Japan. ${ }^{2}$ Department of Stress Protein Processing, Institute of Biomedical and Health Sciences, Hiroshima University, Hiroshima, Japan. ${ }^{3}$ Faculty of Science and Engineering, Kindai University 3-4-1 Kowakae, Higashiosaka City, Osaka, 577-8502, Japan. ${ }^{4}$ Research Institute for Microbial Diseases, Osaka University, 3-1 Yamadaoka, Suita, Osaka, $565-$ 0871, Japan. ${ }^{5}$ Research Institute of Pharmaceutical Sciences, Musashino University, 1-1-20 Shinmachi, Nishi-Tokyo, 202-8585, Japan. ${ }^{6}$ Laboratory of Vaccine Materials and Laboratory of Gut Environmental System, National Institutes of Biomedical Innovation, Health and Nutrition (NIBIOHN), Osaka, 567-0085, Japan. Correspondence and requests for materials should be addressed to A.W. (email: akihiro@phs.osaka-u.ac.jp) or M.K. (email: masuo@phs.osaka-u. ac.jp) 
Furthermore, C-CPE promotes the absorption of human parathyroid hormone, not only via the intestine, but also via the nasal and pulmonary routes ${ }^{16}$. Therefore, modulation of claudin- 4 is a promising strategy for enhancing drug absorption by the paracellular permeation route. However, C-CPE is derived from a toxin fragment, and its immunogenicity may increase with multiple administrations ${ }^{17}$. In addition, it is desirable to develop a low-molecular-weight claudin binder that can be mass-produced at a low cost.

Here, we constructed a high-throughput screening system based on the Time-Resolved Fluorescence Resonance Energy Transfer (TR-FRET) method for identifying claudin-4 binders to regulate mucosal TJ-barrier function ${ }^{18}$. We then used a TR-FRET-based assay to screen 32,560 compounds. The screen identified thiostrepton as a novel claudin-4 binder that attenuates TJ-barrier function in vitro and in vivo. We expect that the TR-FRET-based screening assay will be useful for identifying compounds that modulate the permeability of the mucosal epithelial barrier.

\section{Results}

Preparation of detection assay for claudin-4 and C-CPE interaction by TR-FRET. C-CPE is highly active in lowering TJ barrier function, suggesting that (a) the region of interaction between C-CPE and claudin- 4 is pivotal in the disruption of claudin-4-mediated TJ barrier formation; and (b) interactions between other claudin- 4 binders and this region have the potential to disrupt $\mathrm{TJ}$ barriers and enhance absorption via the paracellular route. To construct a detection system for interaction between claudin- 4 and C-CPE, we prepared purified His (polyhistidine)-tagged claudin-4 (His-claudin-4) and GST (glutathione-S-transferase)-tagged C-CPE (GST-C-CPE) (Supplementary Figure 1) and performed an ELISA to confirm the interaction between His-claudin-4 and GST-C-CPE. By using a range of concentrations of GST-C-CPE, a dose-dependent signal was observed; in contrast, the negative control GST-C-CPE Y306A/L315A showed negligible affinity for claudin- $4^{19}$ (Supplementary Figure 2), indicating that His-claudin-4 interacted with GST-C-CPE.

We then developed a novel TR-FRET-based binding assay to detect interaction between claudin-4 and C-CPE. In this assay, europium $(\mathrm{K})$-conjugated-anti-His- antibody $(\mathrm{Eu}(\mathrm{K})$-anti-His $\mathrm{Ab})$, which binds to His-claudin-4, is used as a donor, and XL-665-conjugated-anti-GST-antibody (XL-665-anti-GST Ab), which binds to GST-C-CPE, is used as an acceptor. The formation of a quaternary complex containing $\mathrm{Eu}(\mathrm{K})$-anti-His Ab, XL-665-anti-GST Ab, His-claudin-4, and GST-C-CPE results in fluorescence energy transfer between donor and acceptor. A binder inhibiting the interaction between claudin-4 and C-CPE disrupts the energy transfer between the donor and acceptor, thus reducing the FRET signal (Fig. 1a). We observed that FRET signals increased after the addition of GST-C-CPE but not after the addition of the negative control C-CPE Y306A/L315A (Fig. 1b). Therefore, the assay successfully detected the interaction of His-claudin- 4 with GST-C-CPE. To assess whether the presence of another claudin- 4 binder attenuated the FRET signal, we performed competition assays using C-CPEs and claudin- 4 antibodies that bind to the C-terminal of claudin-4 (intracellular region) or extracellular domain of claudin-4 (extracellular region $)^{12}$, respectively. The antibody recognising the extracellular domain of claudin- 4 reduced TJ-barrier function $^{12}$. Adding C-CPE or the antibody recognising the extracellular domain of claudin- 4 considerably reduced the FRET signal, whereas adding C-CPE Y306A/L315A or the antibody recognising the C-terminal of claudin-4 did not affect the signals (Fig. 1c), indicating that this assay can be used to identify binders that bind to the extracellular domain of claudin- 4 .

TR-FRET-based screening assay for identification of claudin-4 binders. We optimised the TR-FRET assay for high-throughput screening by focusing on the concentrations of DMSO (dimethylsulfoxide), His-claudin-4, and GST-C-CPE and on the order of addition of compounds in the screening steps; changes in the DMSO concentration $(0.1 \%$ to $2 \%)$ did not alter the FRET signals. The concentrations of C-CPE ( 2.5 to $10 \mathrm{nM}$ ) and claudin-4 (2.5 to $10 \mathrm{nM})$ that, when combined, yielded the highest FRET signal were each $10 \mathrm{nM}$. The number of procedural steps and final reaction time that achieved the optimal signal-to-background ratio and $Z^{\prime}$-factor value were a 4-step assay and 30 min (Supplementary Figure 3).

We used the optimised protocol to screen a 32,560-compound library including (a) the Representative Diversity Set (20,000 compounds), (b) the Pharmacology Diversity set (10,240 compounds), and (c) the Spectrum Collection (2,320 compounds). The assay yielded excellent statistics: the mean $Z^{\prime}$-factor was $0.86 \pm 0.09$, and the mean signal-to-background ratio was $6.9 \pm 1.18$ (Fig. 2a). The 'hit' cutoff was set at $>60 \%$ inhibition of FRET signal, thus corresponding to 67 compounds. We then conducted a cytotoxicity assay of the hit compounds in human Caco-2 cells and identified 6 compounds with possible cytotoxicity. To determine whether the hit compounds bound directly to claudin-4 protein, not C-CPE, we performed Biacore analysis using recombinant human claudin-4 protein; 29 of the 67 compounds exhibited a Resonance Unit value of $>3$, thus indicating direct binding to claudin- 4 . Of the 29 compounds identified in our screen, 4 decreased the transepithelial electrical resistance (TER) value in a Caco- 2 cell monolayer (Supplementary Figure 4). These results indicate that the claudin- 4 binder screening system based on TR-FRET might be a useful tool for identifying claudin- 4 binders with epithelial-barrier-disrupting activity.

Among the hit compounds, thiostrepton achieved the greatest attenuation of the barrier function of Caco- 2 cells (Supplementary Figure 4). Thiostrepton inhibited the interaction between His-claudin-4 and GST-C-CPE at an IC 50 (i.e. the concentration at which interaction is reduced by $50 \%$ ) of $1.017 \mu \mathrm{M}$ (Fig. 2b). Biacore analysis of the interaction between claudin- 4 and thiostrepton revealed a $\mathrm{K}_{\mathrm{D}}$ of $6.615 \times 10^{-6}$ (Supplementary Figure 5). In contrast, thiostrepton did not bind to $\mathrm{C}-\mathrm{CPE}$. To investigate whether thiostrepton prevents $\mathrm{C}-\mathrm{CPE}$ from binding to claudins on the cell membrane, we conducted a competition assay. Thiostrepton inhibited the binding of C-CPE to claudin- 4 on the cell membrane (Fig. 2c). Thiostrepton also inhibited the interaction between C-CPE and claudin-3, but this effect was substantially weaker than that for claudin-4. These results suggest that thiostrepton preferentially binds to claudin- 4 on the cell membrane. 
a

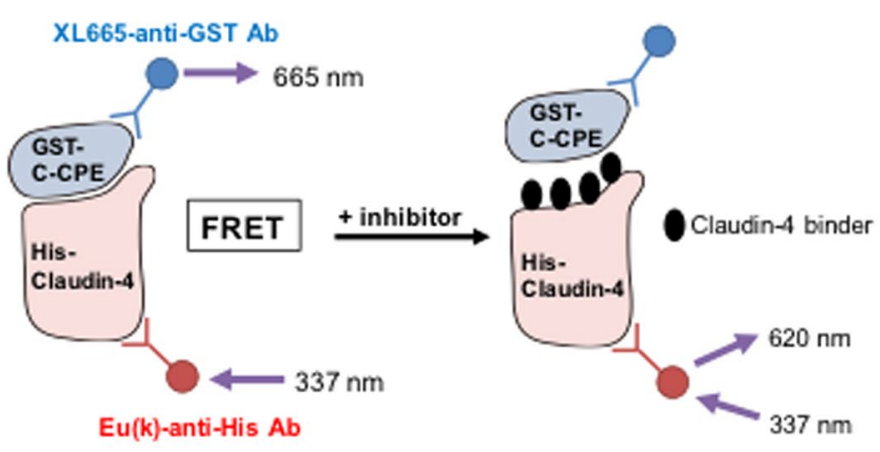

b

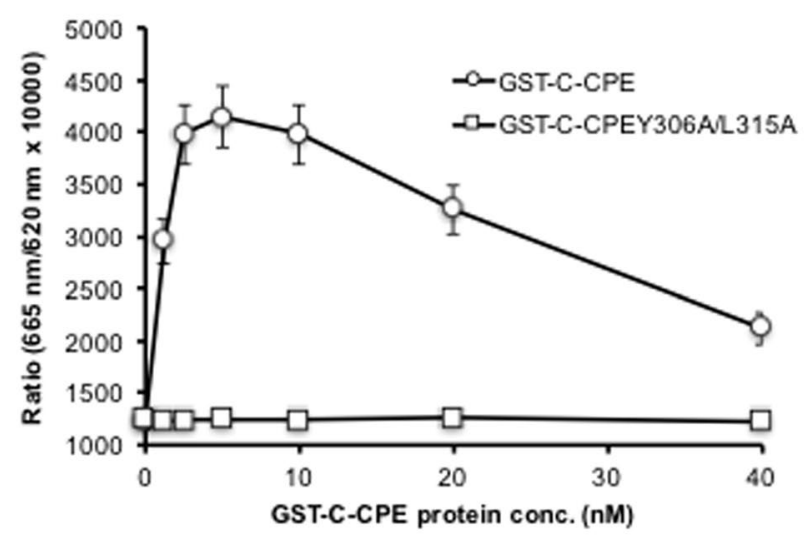

C

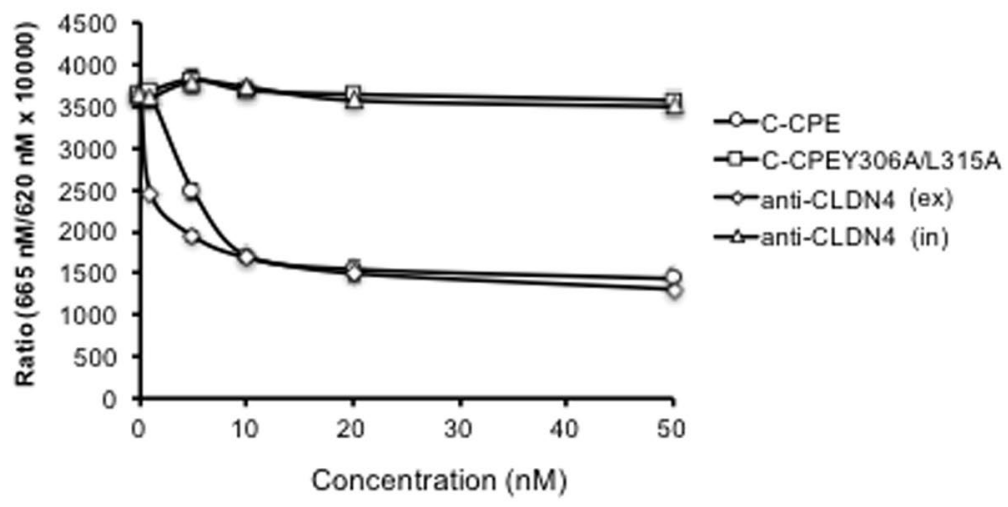

Figure 1. TR-FRET assay for detecting interaction between claudin-4 and C-CPE. (a) Schematic representation of the TR-FRET-based assay for detecting the interaction between claudin- 4 and C-CPE. When claudin- 4 binder binds to the site of claudin-4 and C-CPE interaction, FRET signal is not detected. (b) Validation of the TR-FRET-based claudin- 4 and C-CPE binding assay. The assay was performed by sequentially adding europium (K)-anti-His antibody (Ab), His-tagged claudin-4 (His-claudin-4) and XL665-anti-GST Ab, followed by the addition of GST-tagged C-CPE or C-CPE Y306A/L315A. TR-FRET signals were measured with an Artemis HTRF plate reader at 620 and $665 \mathrm{~nm}$ emission wavelengths. Data are means $\pm S D(n=3)$. (c) Inhibitory effect of claudin- 4 binders on the interaction between His-claudin- 4 and GST-C-CPE in the TR-FRET-based assay. C-CPE, C-CPE Y306A/L315A or anti-claudin-4 Ab (either against the extracellular domain [ex] or the intracellular domain [in] of claudin 4) was incubated with His-claudin-4, and then GST-C-CPE, XL665-anti $\mathrm{GST} \mathrm{Ab}$ and $\mathrm{Eu}(\mathrm{K})$-anti His $\mathrm{Ab}$ were sequentially added. Data are means $\pm \mathrm{SD}(\mathrm{n}=3)$.

Thiostrepton attenuates the barrier function of the intestinal epithelial cell monolayer. Thiostrepton is an antibiotic derived from several strains of streptomycetes ${ }^{20}$, but its influence on the $\mathrm{TJ}$ barrier is unclear. Therefore, to examine the effect of thiostrepton on the barrier function of epithelial cell monolayers, we treated Caco- 2 cells with thiostrepton. Thiostrepton $(1-100 \mu \mathrm{M})$ reversibly decreased the TER value of Caco- 2 cells in a time- and dose-dependent manner (Fig. 3a). We next investigated the effect of thiostrepton on the paracellular permeability of Caco-2 cells by using a paracellular tracer flux assay, with $4-\mathrm{kDa}$ fluorescein isothiocyanate (FITC)-labelled dextran (FD-4) as the tracer. Caco-2 cells were treated with thiostrepton for $24 \mathrm{~h}$, after which 
a

Results of high-throughput screening

\begin{tabular}{l|r|}
\hline Library & $\begin{array}{r}\text { Representative Diversity Set }(20,000 \text { compounds) } \\
\text { Phamaoology Diversity Set (10,240 compounds) } \\
\text { The Soectrum Collection }(2,320 \text { compounds) }\end{array}$ \\
\hline Z'-factor & $0.85 \pm 0.09$ (Average) \\
\hline S/B ratio & $6.90 \pm 1.18$ (Average) \\
\hline Hit compounds & 67 compounds \\
\hline Cytotoxicity test & 6 compounds \\
\hline Biacore test & 29 compounds \\
\hline
\end{tabular}

a Compounds that cause $>60 \%$ inhibition of FRET signal.

'Compounds that result in a survival rate of $<80 \%$ \%

"Compounds with validated interaction with claudin- 4 by biaccre anslysis (resconance units, >3)

b

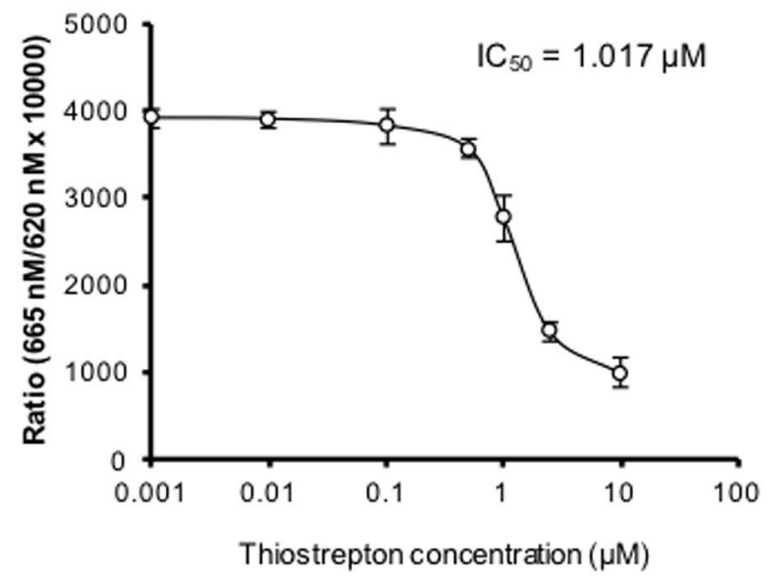

C
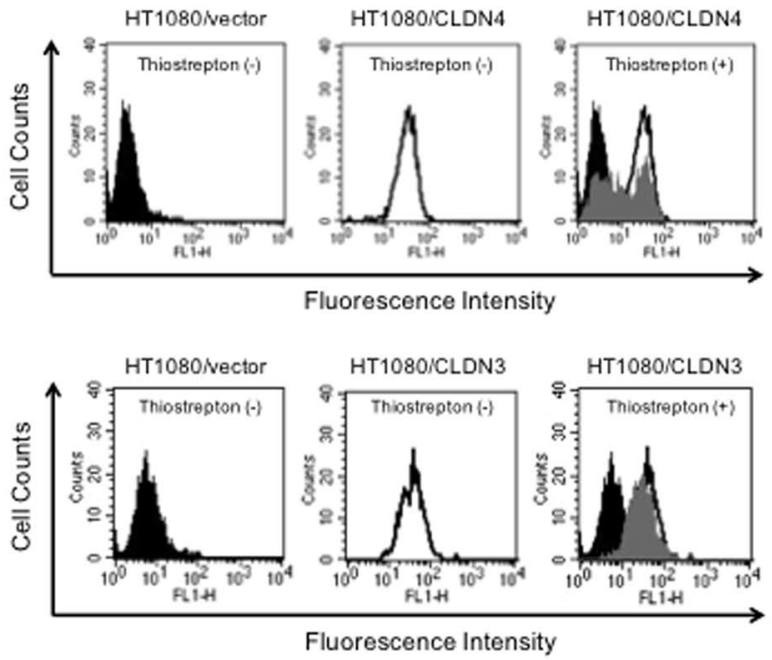

Figure 2. Identification of thiostrepton as a claudin-4 binder. (a) Results of the TR-FRET-based high-throughput screening for claudin- 4 binders. (b) Inhibition of the interaction between claudin- 4 and C-CPE by thiostrepton in the TR-FRET assay. Thiostrepton was assayed in triplicate at various concentrations ( $1 \mathrm{nM}$ to $10 \mu \mathrm{M})$ to assess its inhibitory effect. Data are means $\pm \mathrm{SD}(\mathrm{n}=3)$. $\mathrm{IC}_{50}$, concentration at which the inhibitory response was reduced to $50 \%$. (c) Competition assay of the interaction of thiostrepton with claudin-3 and claudin-4. Claudin-3- or claudin-4expressing HT1080 cells (HT1080/CLDN3 or HT1080/CLDN4) and control (claudin non-expressing) HT1080 cells (HT1080/vector) were treated with vehicle alone, $10 \mu \mathrm{g} / \mathrm{mL}$ BSA or $10 \mu \mathrm{g} / \mathrm{mL}$ BSA plus $1 \mathrm{mM}$ thiostrepton for $1 \mathrm{~h}$. Then, FITC-conjugated C-CPE was added to the cells at $20 \mu \mathrm{g} / \mathrm{mL}$ for $1 \mathrm{~h}$. The thiostrepton-bound cells were used for fluorescence-activated cell sorting analysis. Results for cells treated with vehicle alone are displayed in black. Results for cells treated with BSA with or without thiostrepton are displayed in grey or white, respectively. 
a

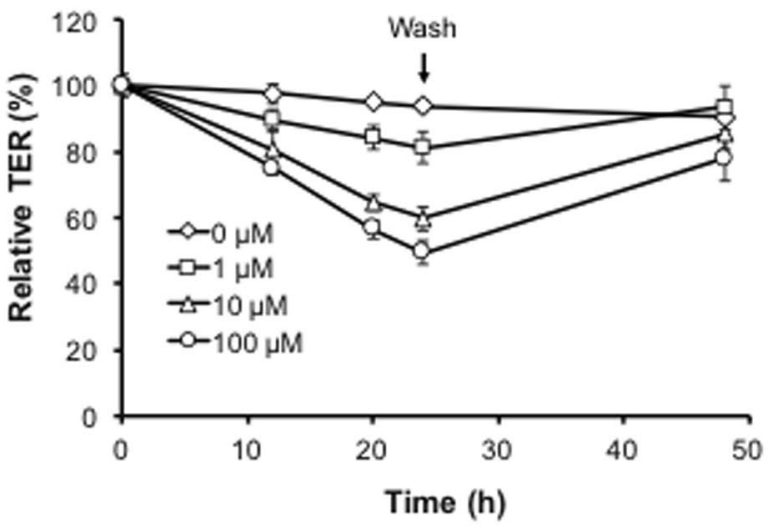

b

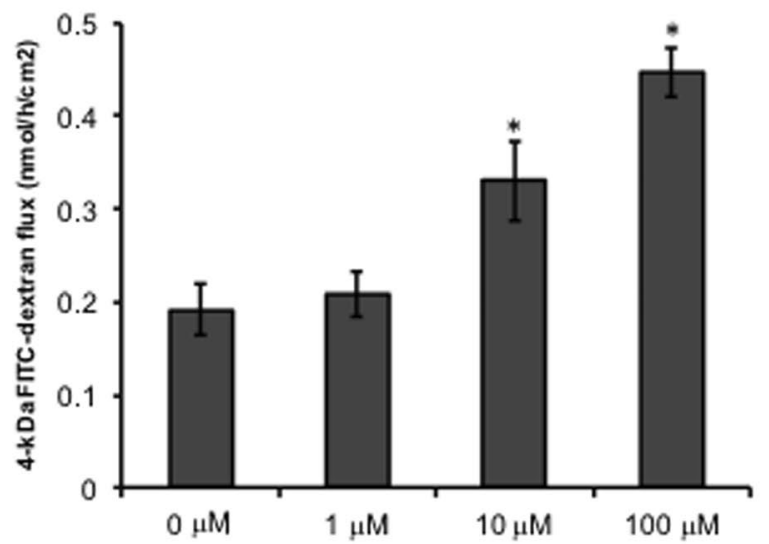

C

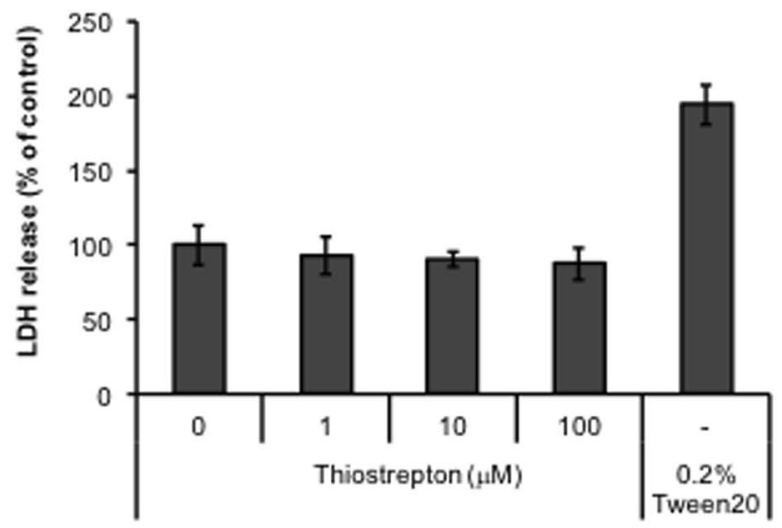

Figure 3. Effect of thiostrepton on the epithelial barrier in a Caco-2 cell monolayer. Caco- 2 cell monolayers were incubated with vehicle or 1,10 , or $100 \mu \mathrm{M}$ thiostrepton. (a) After $24 \mathrm{~h}$, the media were replaced with fresh medium, and the cells were cultured for an additional $24 \mathrm{~h}$. TER values were monitored at 12, 18, 24 and $48 \mathrm{~h}$; TER values are shown as a percentage of the TER value at $0 \mathrm{~h}$. Data are means $\pm \operatorname{SD}(n=3)$. (b) The permeability for 4 -kDa FITC-dextran was measured at $24 \mathrm{~h}$. Data are means $\pm \mathrm{SD}(\mathrm{n}=3)$. $* P<0.05$ vs vehicle-treated group, as determined by using Dunnett's test. (c) The viability of cells treated with thiostrepton for $24 \mathrm{~h}$ was assessed by measuring the level of lactate dehydrogenase $(\mathrm{LDH})$ released into the culture medium. Tween $20(0.2 \%)$ served as the positive control. LDH release is shown as a percentage of the amount released by the vehicle-treated cells. Data are means $\pm S D(n=3)$.

we measured the amount of FD-4 that had passed from the apical side to the basal side. Consistent with the TER decrease, thiostrepton $(1-100 \mu \mathrm{M})$ treatment induced a dose-dependent increase in the paracellular flux of FD-4 (Fig. 3b). Furthermore, thiostrepton was not cytotoxic to Caco-2 cells in the concentration range of 1-100 $\mu \mathrm{M}$ (Fig. 3c). These results indicate that thiostrepton attenuated the TJ-barrier function of Caco-2 cell monolayers. 
a

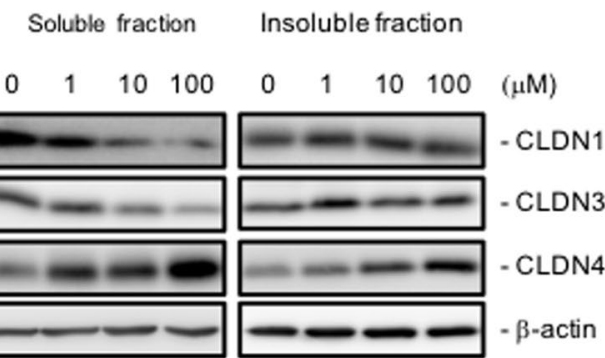

b
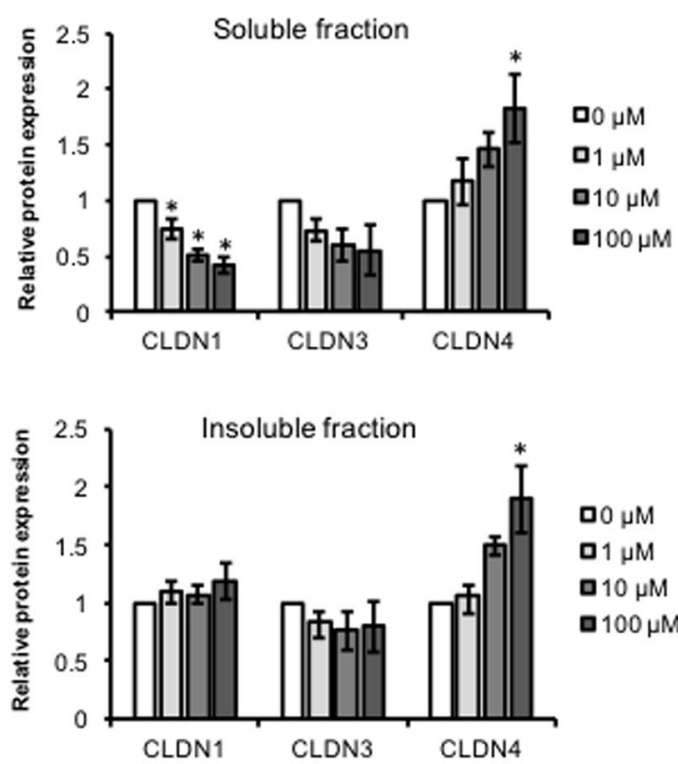

$01 \mu \mathrm{M}$

व $10 \mu \mathrm{M}$

- $100 \mu \mathrm{M}$
C

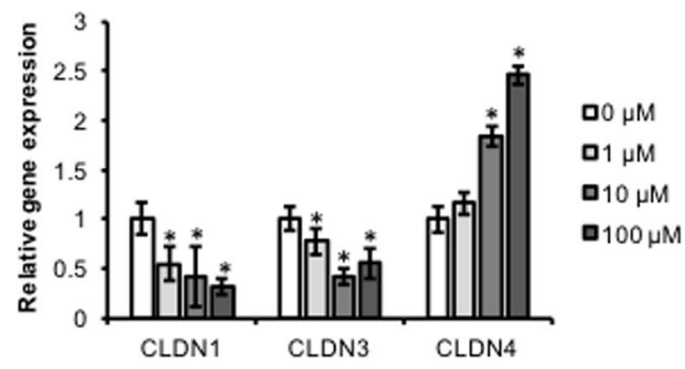

d

Control

Thiostrepton

CLDN1
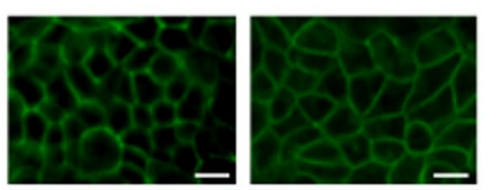

OCLN
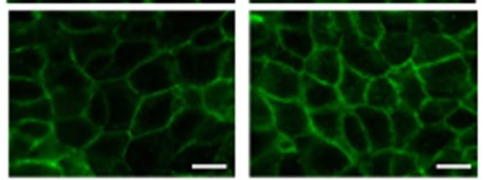

CLDN4
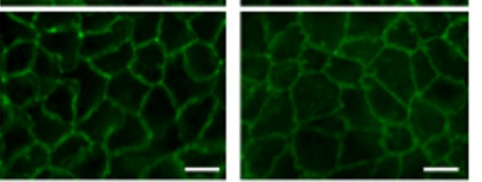

ZO-1
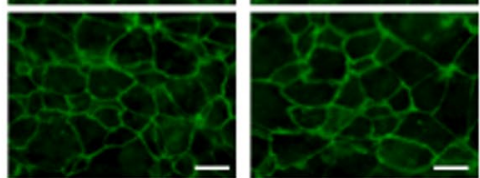

Figure 4. Thiostrepton effect on tight junction components of Caco-2 cell monolayers. Caco-2 cell monolayers were incubated with vehicle or 1,10 or $100 \mu \mathrm{M}$ thiostrepton. Triton-X (1\%)-soluble and -insoluble lysates were collected and immunoblotted for claudin-1 (CLDN1), claudin-3 (CLDN3) or claudin-4 (CLDN4) (a). $\beta$-actin served as the loading control. Pre-cropped blots are included in Supplementary Figure 6. Relative protein density was calculated as the ratio of the protein density to the density in the vehicle control (b). Data are means $\pm \mathrm{SD}(\mathrm{n}=3) . * P<0.05$ vs. vehicle-treated group, as determined by using Dunnett's test. (c) Caco-2 cells were treated with or without $100 \mu \mathrm{M}$ thiostrepton for $24 \mathrm{~h}$, and then cell lysates were subjected to qRTPCR analysis for CLDN1, CLDN3, or CLDN4. Data are means $\pm S D(n=3)$. $* P<0.05$ vs vehicle-treated group, as determined by using Dunnett's test. (d) Immunofluorescence localisation of CLDN1, CLDN4, occludin (OCLN) and ZO-1 after treatment with $100 \mu \mathrm{M}$ thiostrepton or no treatment for $24 \mathrm{~h}$. Bar $=10 \mu \mathrm{m}$.

Effect of thiostrepton on TJ proteins. To investigate the effect of thiostrepton on TJ components of Caco- 2 cells, we separated the $1 \%$ Triton-X-soluble and -insoluble fractions and performed immunoblotting and qRT-PCR analyses of cell-cell adhesion molecules. Thiostrepton treatment significantly decreased the $1 \%$ Triton-X-soluble protein and mRNA levels of claudin-1 and claudin-3 (Fig. 4a-c and Supplementary Figure 6), whereas the protein levels in the $1 \%$ Triton-X-insoluble fraction were unchanged. In contrast, thiostrepton treatment increased the $1 \%$ Triton-X-soluble protein and mRNA levels, and the $1 \%$ Triton-X-insoluble protein levels, of claudin-4. Immunofluorescence staining showed that thiostrepton treatment did not alter the cellular localisation of claudin-1, claudin-4, occludin or ZO-1 (Fig. 4d). To evaluate the effect of thiostrepton on the paracellular permeation of molecules, we used sulfo-NHS-SS-biotin $(607 \mathrm{Da})$, which is a cell-membrane-impermeable molecule ${ }^{21,22}$. When Caco- 2 cell monolayers were treated with thiostrepton and then apically incubated with the biotin compound, biotin signal was detected within the paracellular space; in contrast, no biotin permeation was observed in untreated control cultures (Fig. 5). The signal in the control cultures was due to residual biotin that remained on the apical side after washing; it was not translocated biotin. These results suggest that thiostrepton attenuates TJ-barrier function without distinct changes in the localisation of the TJ components.

Thiostrepton enhances intestinal absorption of a macromolecule. To investigate the effect of thiostrepton on the jejunal absorption of a macromolecule, we conducted an in situ loop assay, using FD-4 as the macro-tracer molecule. Thiostrepton enhanced rat jejunal absorption of FD-4 in a dose-dependent manner, as 
Control

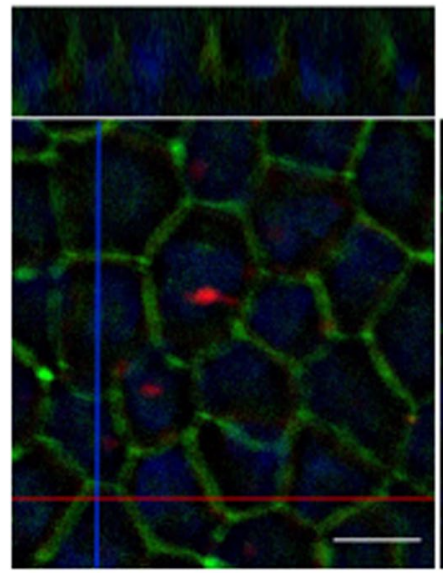

Thiostrepton

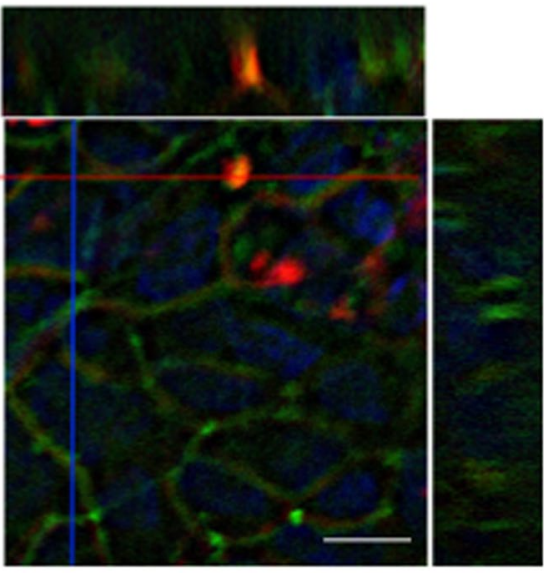

Figure 5. Thiostrepton effect on paracellular tracer permeability. Caco-2 cell monolayers were treated with $100 \mu \mathrm{M}$ thiostrepton or left untreated (control) and then apically incubated with sulfo-NHS-SS-biotin (606.7 Da; red) and counterstained with anti-ZO-1 antibody (green; TJ stain) and DAPI (blue; nuclear stain). Scanning of the lateral ( $\mathrm{z}-$ ) axis of the cells showed specific localisation of the biotin signals (red) within intercellular contacts, whereas no biotin permeation was observed in control cells. Bar $=10 \mu \mathrm{m}$.

compared with that in the vehicle-treated group (Fig. 6a and b). In addition, thiostrepton treatment increased plasma FD-4 concentrations, which reached a maximum of $0.6 \mu \mathrm{g} / \mathrm{mL}$. Histochemical analysis showed no evidence of intestinal tissue toxicity (Fig. 6c and Supplementary Figure 7). These results indicate that thiostrepton has the potential to enhance the intestinal absorption of macromolecules without intestinal toxicity.

\section{Discussion}

Although several claudin-4 binders have been reported ${ }^{23-25}$, C-CPE and its variants are the most commonly reported claudin- 4 binders known to regulate TJ barrier function ${ }^{8,26-28}$. C-CPE has the potential to enhance the absorption of macromolecule-like peptides in various mucosal tissues ${ }^{16,29}$. Therefore, modulation of claudin4-based TJ-barrier function is a promising strategy for developing an absorption enhancer. However, a screening system for identifying claudin binders that might regulate TJ-barrier function had not been developed previously. Here, we constructed a claudin-4 binder screening system based on TR-FRET, which detects the interaction between claudin-4 and C-CPE. We surmise that our novel screening system will not only simplify the identification of compounds binding to claudin- 4 but will also restrict the outcome to compounds with TJ barrier-regulating activity, given that the compounds obtained bind substantially to the claudin-4 region that interacts with C-CPE. In fact, we showed that TR-FRET analysis distinguished between an antibody that recognises the intracellular domain of claudin-4, which cannot regulate TJ barriers, and one that recognises the extracellular domain of claudin-4, which attenuates TJ-barrier function. By using this system, we successfully identified novel claudin- 4 binders that attenuated epithelial barrier function. Among them, thiostrepton strongly attenuated the TJ-barrier function of Caco- 2 cell monolayers. Furthermore, we demonstrated that thiostrepton enhanced rat jejunal absorption of a macromolecule without tissue toxicity. Therefore, our screening system is appropriate for identifying TJ-modulating claudin-4 binders.

Thiostrepton is a veterinary antibiotic used to treat dermatological diseases and mastitis caused by Gram-negative bacteria ${ }^{20,30}$. In addition, thiostrepton shows anti-tumour activity in various human cancer cell lines ${ }^{31-35}$; in melanoma cells, thiostrepton suppresses cell growth and induces apoptosis ${ }^{36,37}$; and in breast cancer cells, thiostrepton leads to cell-cycle arrest and cell death by down-regulating FOXM1 gene expression ${ }^{38}$. Here, we demonstrated that thiostrepton can attenuate intestinal TJ-barrier function and increase mucosal absorption of macromolecules, indicating that thiostrepton has absorption-enhancing properties. Biacore analysis revealed that thiostrepton directly bound to claudin- 4 with a $\mathrm{K}_{\mathrm{D}}$ of $6.615 \times 10^{-6}$. Competition analysis showed that thiostrepton bound to claudin- 4 in the cellular membrane, indicating that claudin- 4 is a target molecule for thiostrepton. Thiostrepton also bound to claudin-3 in the cellular membrane, although the affinity for claudin-3 was considerably lower than for claudin-4. C-CPE binds not only to claudin-4 but also to other claudins, including claudins-3, $-6,-7$, and $-9^{39}$. These findings suggest that the screening system may not completely eliminate, as hit compounds, molecules that bind to other claudins.

TER and tracer flux assays revealed that thiostrepton increased the paracellular permeability of Caco-2 cell monolayers without inducing cell toxicity. Indeed, thiostrepton treatment induced the passage of visibly labelled biotin via the paracellular route. In contrast, the localisation of TJ components, such as claudin-1, claudin-4, occludin and ZO-1, did not change after thiostrepton treatment. These results suggest that the thiostrepton-induced attenuation of barrier function was not due to disruption of the TJ complex but rather perhaps to the disruption of claudin-claudin interactions formed between adjacent cells. Although thiostrepton reduced the TJ-barrier function of Caco- 2 cells, it promoted the transcriptional expression and membrane 
a

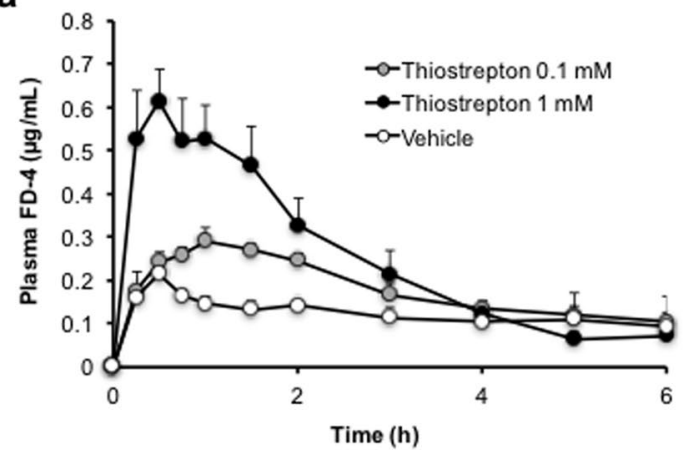

b

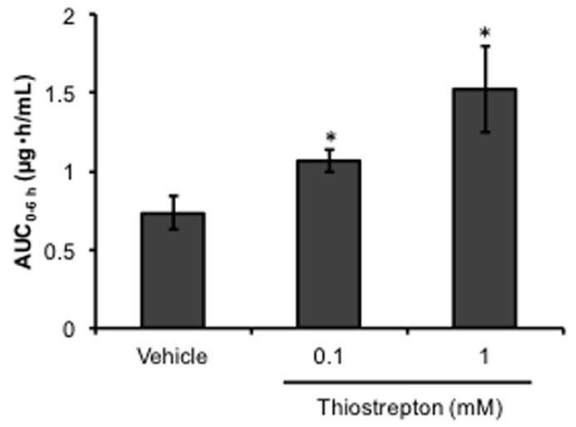

C

Vehicle

Thiostrepton

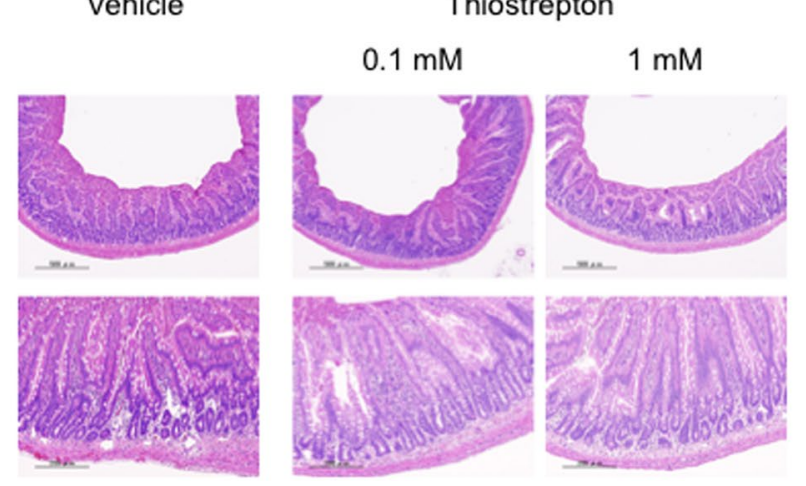

Figure 6. Mucosal absorption-enhancing effect of thiostrepton. (a) Rat jejunum was treated with vehicle alone or with 0.1 or $1 \mathrm{mM}$ thiostrepton and $1 \mathrm{mg} 4$-kDa FITC-labelled dextran (FD-4). Time-course changes in plasma FD-4 concentration were monitored, and the area under the time-concentration curve between 0 and $6 \mathrm{~h}\left(\mathrm{AUC}_{0-6 \mathrm{~h}}\right)$ was calculated $(\mathbf{b})$. Data are means $\pm \mathrm{SD}(\mathrm{n}=4) . * P<0.05$ vs vehicle-treated group, as determined by using Dunnett's test. (c) Histological analysis of representative thiostrepton-treated rat jejunum. After $6 \mathrm{~h}$ of treatment with vehicle or thiostrepton $(0.1$ or $1 \mathrm{mM})$, the jejunum was fixed and stained with haematoxylin and eosin.

protein production of claudin-4. One possible explanation for this inconsistency may be a reaction to restore the attenuated claudin-4 function. Alternatively, thiostrepton treatment decreased the transcription and cytoplasmic protein production of claudins- 1 and -3 , whereas the amounts of these proteins in the membrane fraction, which is involved in TJ-barrier function, were not altered. The differences in claudin production levels might be involved with the interaction of thiostrepton with other claudins. The mechanisms underlying the thiostrepton-induced production of claudins are unclear, and future studies need to investigate the effect of thiostrepton on TJ function.

Thiostrepton represents a novel and effective permeability enhancer in intestinal epithelial cells. Partial modification of the structure of thiostrepton might improve its binding properties and specificity. The recent reporting of the structure of the claudin-4-C-CPE complex ${ }^{40}$ will probably facilitate such studies ${ }^{41}$. Although claudin- 4 plays a major role in mucosal TJ barriers, its production increases in many malignant tumour types ${ }^{42}$, and it is produced at high levels in epithelial tissues covered with mucosal immune tissues ${ }^{43,44}$. Several reports have indicated that claudin-4 binders are effective in treating cancer (e.g., pancreatic, ovarian, breast, colorectal and gastric cancer cells $)^{23,24,45-47}$ and can be delivered as a vaccine to mucosal immune tissue ${ }^{44,48,49}$. Therefore, the preparation of an effective claudin- 4 binder may help to reveal new pharmacological strategies to improve drug delivery, cancer treatment and mucosal vaccine development.

\section{Methods}

Reagents, antibodies and cells. n-Dodecyl- $\beta$-D-maltoside (DDM) was purchased from Dojindo Laboratories (Kumamoto, Japan). FD-4 was purchased from Sigma-Aldrich (St. Louis, MO, USA). Monoclonal anti-6 histidine antibody conjugated europium ${ }^{3+}$ cryptate $(\mathrm{Eu}(\mathrm{K})$-anti-His $\mathrm{Ab})$ and monoclonal anti-GST antibody labelled with XL665 (XL665-anti-GST Ab) were purchased from CIS Bio International (Gif-sur-Yvette, France). Rabbit anti-claudin-1 polyclonal antibody (pAb), rabbit anti-claudin-3 pAb, mouse anti-claudin-4 monoclonal $\mathrm{Ab}(\mathrm{mAb})$, mouse anti-occludin $\mathrm{mAb}$ and rabbit anti-ZO-1 $\mathrm{pAb}$ were purchased from Invitrogen (Carlsbad, CA, USA). Mouse anti- $\beta$-actin $\mathrm{mAb}$ was purchased from Sigma-Aldrich. Goat anti-rabbit IgG peroxidase-conjugated antibody and goat anti-mouse IgG peroxidase-conjugated antibody were purchased from Millipore (Bedford, MA, USA). Alexa Fluor 488 goat anti-rabbit IgG and Alexa Fluor 488 goat anti-mouse IgG were purchased from Molecular Probes (Eugene, OR, USA). 
The human colorectal adenocarcinoma cell line Caco-2 (HTB-37) was obtained from the American Type Culture Collection (Rockville, MD, USA). Caco-2 cells were cultured in Eagle's minimum essential medium (Nissui, Japan) supplemented with $10 \%$ foetal bovine serum under $5 \% \mathrm{CO}_{2}$ at $37^{\circ} \mathrm{C}$. The passage number of the cells used for the experiments was between 10 and 20 .

Preparation of GST-C-CPEs and C-CPEs. To prepare glutathione-S-transferase-fused C-CPEs (GST-C-CPEs), plasmids encoding C-CPE or C-CPE Y306A/L315A were cloned into pGEX-4T-1 (GE Healthcare, Munich, Germany). The resultant plasmids were transduced into Escherichia coli strain BL21 (DE3), after which the cells were cultured in Terrific Broth medium at $37^{\circ} \mathrm{C}$. Isopropyl-1-thio- $\beta$-D-galactoside was then added to the cultures, and the cells were grown for an additional $3 \mathrm{~h}$. The cells were solubilised in STE buffer $(10 \mathrm{mM}$ Tris- $\mathrm{HCl}, 150 \mathrm{mM} \mathrm{NaCl}, 1 \mathrm{mM}$ ethylenediaminetetraacetic acid $\mathrm{pH} 8.0)$ containing $10 \mu \mathrm{g} / \mathrm{mL}$ lysozyme, $5 \mathrm{mM}$ dithiothreitol and $1.5 \% \mathrm{~N}$-lauroylsarcosine. The lysates were centrifuged, and the supernatants were collected and adjusted to $2 \%$ Triton X-100. The supernatants were incubated with glutathione-Sepharose beads (GE Healthcare) for $3 \mathrm{~h}$ at $4{ }^{\circ} \mathrm{C}$, the beads were washed with STE buffer, and then the GST-C-CPEs were eluted from the beads with STE buffer containing $25 \mathrm{mM}$ glutathione. For preparation of C-CPEs, C-CPEs were cleaved from the GST-C-CPEs with thrombin (Nacalai Tesque, Kyoto, Japan). The solvents were exchanged with phosphate-buffered saline (PBS) by using a PD-10 column (GE Healthcare).

Preparation of claudin-4 protein. Recombinant human claudin-4 protein was prepared by an expression system using Sf9 cells and recombinant baculovirus ${ }^{16}$. Briefly, the C-terminal His-tagged claudin- 4 cDNA fragment was cloned into pFastBacl (Invitrogen), and recombinant baculovirus was generated by using the Bac-to-Bac baculovirus expression system (Invitrogen). Sf9 cells were infected with the recombinant baculovirus. After $72 \mathrm{~h}$ of the infection, the cells were suspended in PBS supplemented with protease inhibitor cocktail (Nacalai Tesque) and 20 units/mL DNase I. The cells were lysed by addition of DDM (to a final concentration of $2 \%$ ). The resultant supernatant was applied to a HisTrap HP column (GE Healthcare), and His-claudin-4 was eluted with $500 \mathrm{mM}$ imidazole. The solvent for His-claudin- 4 was exchanged to PBS containing $0.2 \%$ DDM by using a PD-10 column (GE Healthcare).

TR-FRET assay. In the TR-FRET assay, the $\mathrm{Eu}(\mathrm{K})$-anti-His $\mathrm{Ab}$, which recognises His-claudin-4, was the donor molecule, and the XL665-anti-GST Ab, which recognises GST-C-CPEs, was the acceptor molecule. When His-claudin-4 and GST-C-CPEs were in close proximity, a transfer of fluorescence resonance energy between the donor and acceptor molecules resulted. The assay was performed in a final volume of $100 \mu \mathrm{L} /$ well in 96 -well plates. $\mathrm{Eu}(\mathrm{K})$-anti-His $\mathrm{Ab}(0.125 \mathrm{nM})$, His-claudin-4 (10 nM) and XL665-anti-GST Ab (10 nM) were dissolved in TR-FRET buffer ( $100 \mathrm{mM} \mathrm{KF}$ and $0.1 \%$ bovine serum albumin [BSA] in PBS) and added to the 96 -well plates. Then, GST-C-CPEs (0-40 nM) were added and mixed. After $30 \mathrm{~min}$ of incubation at room temperature, FRET signals were measured with an Artemis HTRF plate reader (Berthold Technologies, Wildbad, Germany).

For high-throughput screening, the TR-FRET assay was modified to a 384-well plate format (Supplementary Figure 3). The screening assay was performed in a final volume of $25 \mu \mathrm{L} /$ well. His-claudin- $4(10 \mathrm{nM})$ and library compounds $(10 \mu \mathrm{M})$ were dissolved in TR-FRET buffer and incubated for $1 \mathrm{~h}$. Then, GST-C-CPE $(10 \mathrm{nM})$, XL665-anti-GST Ab $(10 \mathrm{nM})$ and $\mathrm{Eu}(\mathrm{K})$-anti-His $\mathrm{Ab}(0.125 \mathrm{nM})$ were sequentially added after each 30 -min incubation. FRET signals were measured with an Artemis HTRF plate reader (Berthold Technologies). Library compounds (Representative Diversity Set [20,000 compounds], Pharmacology Diversity set [10,240 compounds] and the Spectrum Collection [2,320 compounds]) were obtained from the Platform for Drug Discovery (Osaka University, Osaka, Japan); these libraries are also commercially available. Detailed information about the libraries can be accessed at their respective web sites: http://www.namiki-s.co.jp/service/screening/kit_product.html and http://www.msdiscovery.com/spectrum.html.

Flow cytometry analysis. Claudin-3 or claudin-4-producing HT1080 cells were incubated with thiostrepton $(1 \mathrm{mM})$ for $1 \mathrm{~h}$ at $4^{\circ} \mathrm{C}$, washed with $1 \%$ BSA in PBS, and then incubated with FITC-labelled C-CPE for $1 \mathrm{~h}$ at $4^{\circ} \mathrm{C}$. After being washed with $1 \%$ BSA in PBS, the cells were analysed by flow cytometry (FACS Calibur, Becton Dickinson, Franklin Lakes, NJ, USA).

Barrier assays. Caco-2 cells were allowed to differentiate for 10 to 14 days on Transwell filters (diameter, 6.5 $\mathrm{mm}$; pore size, $0.4 \mu \mathrm{m}$; Corning, Corning, MA, USA) before their use in TER and paracellular flux assays. Caco-2 cell monolayers had TER values of $600-800 \Omega \bullet \mathrm{cm}^{2}$. TER values were measured by using a Millicell-ERS epithelial volt-ohm meter (Millipore Corporation, Billerica, MA, USA). For the paracellular tracer flux assay, $100 \mu \mathrm{M}$ dialysed FD- 4 was added to the medium in the upper chamber. After $1 \mathrm{~h}$ of incubation, the medium was collected from the bottom chamber, and the amount of FD-4 was measured with a TriStar LB 941 microplate reader (Berthold Technologies).

Lactate dehydrogenase release assay. For analysis of thiostrepton-induced cytotoxicity on Caco-2 cells, a lactate dehydrogenase (LDH) release assay was performed according to the manufacturer's instructions. After being treated with thiostrepton for $24 \mathrm{~h}$, the level of $\mathrm{LDH}$ released into the culture medium was analysed. Treatment with $0.2 \%$ Tween-20 was used as a $100 \%$ lysis control. Absorbance was measured at a wavelength of $570 \mathrm{~nm}$ by using a TriStar LB 941 microplate reader (Berthold Technologies).

Immunoblotting analysis. Caco-2 cells were cultured on a Transwell filter (diameter, $24 \mathrm{~mm}$; pore size, $0.4 \mu \mathrm{m}$; Corning). To prepare Triton-X-soluble and -insoluble cell lysates, cells were lysed with $1 \%$ Triton-X buffer (50 mM Tris-HCl [pH 7.4], 1.0\% Triton X-100, $5 \mathrm{mM}$ ethylene glycol-bis(2-aminoethylether)-N,N,N', $\mathrm{N}^{\prime}$-tetraacetic acid) containing a protease inhibitor cocktail (Sigma-Aldrich). Cell lysates were centrifuged at 
$15,600 \times g$ for $5 \mathrm{~min}$ at $4{ }^{\circ} \mathrm{C}$ to sediment the high-density, actin-rich fraction. The pellet was re-suspended in cell lysis buffer ( $10 \mathrm{mM}$ Tris- $\mathrm{HCl}$ [pH 7.4], 0.3\% sodium dodecyl sulphate [SDS]) containing protease inhibitor cocktail. The cell lysates were separated on an SDS-polyacrylamide gel and electroblotted onto polyvinylidene difluoride membranes. The membranes were incubated successively with antibodies against claudins- $1,-3$ and -4 and $\beta$-actin and then with a horseradish-peroxidase-conjugated anti-rabbit or anti-mouse IgG antibody. The reactive bands were detected with Chemi-Lumi One (Nacalai Tesque), and the signals were visualised with ImageQuant LAS4010 (GE Healthcare). The corresponding immunoblot bands were analysed with ImageJ.

Quantitative reverse transcription - PCR (qRT-PCR) analysis. Total RNA of Caco-2 cells left untreated or treated with thiostrepton for $24 \mathrm{~h}$ was isolated by using Sepasol-RNA reagent (Nacalai Tesque), and the RNA $(3 \mu \mathrm{g})$ was reverse-transcribed by using oligo $(\mathrm{dT})$ primers and a cDNA synthesis kit (Roche, Mannheim, Germany). Quantitative RT-PCR was performed with SYBR Premix Ex Taq II (Takara Bio, Shiga, Japan) by using an Applied Biosystems StepOne Plus system (Applied Biosystems, Foster City, CA, USA). Relative quantification was performed against a standard curve, and the values were normalised against the input determined for the housekeeping gene glyceraldehyde 3-phosphate dehydrogenase (GAPDH). PCR primers were as described previously ${ }^{50}$.

Immunofluorescence staining. Localisation of claudins- 1 and -4 , occludin and ZO- 1 on Caco- 2 cells was assessed by immunofluorescence microscopy. Caco-2 cells were seeded on Transwell filters (diameter, $12 \mathrm{~mm}$; pore size, $0.4 \mu \mathrm{m}$; Corning). After treatment with thiostrepton $(100 \mu \mathrm{M})$ or no treatment for $24 \mathrm{~h}$, the Caco- 2 cells were fixed with methanol-acetone $(1: 1 \mathrm{v} / \mathrm{v})$ for $5 \mathrm{~min}$ and then permeabilised with $0.2 \%$ Triton X-100 in PBS for $10 \mathrm{~min}$. After non-specific binding had been blocked by incubation with $1 \% \mathrm{BSA}$ in TBS buffer $(20 \mathrm{mM}$ Tris- $\mathrm{HCl}$, pH 7.4, $40 \mathrm{mM} \mathrm{NaCl}$ ) containing $0.05 \%$ Tween-20 (T-TBS) for $1 \mathrm{~h}$, the cells were incubated with anti-claudin-1, -claudin-4, -occludin and -ZO-1 antibodies in 1\% BSA in T-TBS for $1 \mathrm{~h}$. After incubation of the cells with fluorescent secondary antibodies for $1 \mathrm{~h}$, the immunofluorescence images were observed under an immunofluorescence microscope (Keyence, Tokyo, Japan). For visualisation of biotin passing through the TJ, Caco-2 cells treated with $100 \mu \mathrm{M}$ thiostrepton or left untreated were apically labelled with sulfo-NHS-SS-biotin $(606.7 \mathrm{Da})$ for $10 \mathrm{~min}$. The cells were then fixed, and anti-ZO-1 antibody and 4,6-diamidino-2-phenylindole dihydrochloride (DAPI) were employed for immunofluorescence staining of the TJ or nucleus, respectively.

In situ loop assay. All procedures involving mice and experimental protocols were approved by the Ethics Committee of Osaka University (permission no. 27-5-2). Rat jejunal absorption of FD-4 was evaluated by using an in situ loop assay. After 7-week-old Wister male rats were anesthetised, a midline abdominal incision was made and the jejunum was washed with PBS. A 5-cm-long jejunal loop was prepared and both ends were closed with sutures. A mixture of $1 \mathrm{mg}$ FD-4 and 0.1 or $1 \mu \mathrm{M}$ thiostrepton in $100 \mu \mathrm{L}$ was injected into the jejunal loop. Blood was collected at the time points indicated. Plasma levels of FD-4 were measured with a TriStar LB 941 microplate reader (Berthold Technologies). The area under the plasma concentration curve of the FD- 4 from 0 to $6 \mathrm{~h}$ was calculated by using the trapezoidal method. Recovered intestinal tissues were stained with haematoxylin and eosin and examined for tissue toxicity.

Statistical analysis. Data are presented as means \pm 1 SD. Dunnett's test was used for statistical analyses. A $P$ value of less than 0.05 was considered indicative of statistical significance.

\section{References}

1. Goldberg, M. \& Gomez-Orellana, I. Challenges for the oral delivery of macromolecules. Nat Rev Drug Discov 2, 289-295, https:// doi.org/10.1038/nrd1067 (2003).

2. Maher, S., Mrsny, R. J. \& Brayden, D. J. Intestinal permeation enhancers for oral peptide delivery. Adv Drug Deliv Rev 106, 277-319, https://doi.org/10.1016/j.addr.2016.06.005 (2016).

3. Farquhar, M. G. \& Palade, G. E. Junctional complexes in various epithelia. J Cell Biol 17, 375-412 (1963).

4. Anderson, J. M. \& Van Itallie, C. M. Tight junctions and the molecular basis for regulation of paracellular permeability. Am J Physiol 269, G467-475 (1995).

5. Tscheik, C., Blasig, I. E. \& Winkler, L. Trends in drug delivery through tissue barriers containing tight junctions. Tissue Barriers 1, e24565, https://doi.org/10.4161/tisb.24565 (2013).

6. Wong, V. \& Gumbiner, B. M. A synthetic peptide corresponding to the extracellular domain of occludin perturbs the tight junction permeability barrier. J Cell Biol 136, 399-409 (1997)

7. Fasano, A. \& Uzzau, S. Modulation of intestinal tight junctions by Zonula occludens toxin permits enteral administration of insulin and other macromolecules in an animal model. J Clin Invest 99, 1158-1164, https://doi.org/10.1172/JCI119271 (1997).

8. Kondoh, M. et al. A novel strategy for the enhancement of drug absorption using a claudin modulator. Mol Pharmacol 67, 749-756, https://doi.org/10.1124/mol.104.008375 (2005).

9. Song, K. H., Fasano, A. \& Eddington, N. D. Effect of the six-mer synthetic peptide (AT1002) fragment of zonula occludens toxin on the intestinal absorption of cyclosporin A. Int J Pharm 351, 8-14, https://doi.org/10.1016/j.ijpharm.2007.09.011 (2008).

10. Zwanziger, D. et al. A peptidomimetic tight junction modulator to improve regional analgesia. Mol Pharm 9, 1785-1794, https://doi. org/10.1021/mp3000937 (2012)

11. Krug, S. M. et al. Angubindin-1, a novel paracellular absorption enhancer acting at the tricellular tight junction. J Control Release 260, 1-11, https://doi.org/10.1016/j.jconrel.2017.05.024 (2017)

12. Nakajima, M. et al. Claudin-1 Binder Enhances Epidermal Permeability in a Human Keratinocyte Model. J Pharmacol Exp Ther 354, 440-447, https://doi.org/10.1124/jpet.115.225391 (2015).

13. Morita, K., Furuse, M., Fujimoto, K. \& Tsukita, S. Claudin multigene family encoding four-transmembrane domain protein components of tight junction strands. Proc Natl Acad Sci USA 96, 511-516 (1999).

14. Gunzel, D. \& Fromm, M. Claudins and other tight junction proteins. Compr Physiol 2, 1819-1852, https://doi.org/10.1002/cphy. c110045 (2012). 
15. Sonoda, N. et al. Clostridium perfringens enterotoxin fragment removes specific claudins from tight junction strands: Evidence for direct involvement of claudins in tight junction barrier. J Cell Biol 147, 195-204 (1999).

16. Uchida, H. et al. A claudin-4 modulator enhances the mucosal absorption of a biologically active peptide. Biochem Pharmacol 79, 1437-1444, https://doi.org/10.1016/j.bcp.2010.01.010 (2010).

17. Suzuki, H. et al. A toxicological evaluation of a claudin modulator, the C-terminal fragment of Clostridium perfringens enterotoxin, in mice. Pharmazie 66, 543-546 (2011).

18. Ergin, E., Dogan, A., Parmaksiz, M., Elcin, A. E. \& Elcin, Y. M. Time-Resolved Fluorescence Resonance Energy Transfer [TR-FRET] Assays for Biochemical Processes. Curr Pharm Biotechnol 17, 1222-1230 (2016).

19. Takahashi, A. et al. Domain mapping of a claudin-4 modulator, the C-terminal region of C-terminal fragment of Clostridium perfringens enterotoxin, by site-directed mutagenesis. Biochem Pharmacol 75, 1639-1648, https://doi.org/10.1016/j.bcp.2007.12.016 (2008).

20. Bagley, M. C., Dale, J. W., Merritt, E. A. \& Xiong, X. Thiopeptide antibiotics. Chem Rev 105, 685-714, https://doi.org/10.1021/ cr0300441 (2005)

21. Krug, S. M. et al. Sodium caprate as an enhancer of macromolecule permeation across tricellular tight junctions of intestinal cells. Biomaterials 34, 275-282, https://doi.org/10.1016/j.biomaterials.2012.09.051 (2013).

22. Dittmann, I. et al. Laurate permeabilizes the paracellular pathway for small molecules in the intestinal epithelial cell model HT-29/ B6 via opening the tight junctions by reversible relocation of claudin-5. [Corrected]. Pharm Res 31, 2539-2548, https://doi. org/10.1007/s11095-014-1350-2 (2014).

23. Suzuki, M. et al. Therapeutic antitumor efficacy of monoclonal antibody against Claudin- 4 for pancreatic and ovarian cancers. Cancer Sci 100, 1623-1630, https://doi.org/10.1111/j.1349-7006.2009.01239.x (2009).

24. Hashimoto, Y. et al. Efficacy and safety evaluation of claudin-4-targeted antitumor therapy using a human and mouse cross-reactive monoclonal antibody. Pharmacol Res Perspect 4, e00266, https://doi.org/10.1002/prp2.266 (2016).

25. Li, X. et al. Development of an anti-claudin-3 and -4 bispecific monoclonal antibody for cancer diagnosis and therapy. J Pharmacol Exp Ther 351, 206-213, https://doi.org/10.1124/jpet.114.216911 (2014).

26. Liao, Z. et al. Specific binding of a mutated fragment of Clostridium perfringens enterotoxin to endothelial claudin-5 and its modulation of cerebral vascular permeability. Neuroscience 327, 53-63, https://doi.org/10.1016/j.neuroscience.2016.04.013 (2016).

27. Takahashi, A. et al. Mutated C-terminal fragments of Clostridium perfringens enterotoxin have increased affinity to claudin-4 and reversibly modulate tight junctions in vitro. Biochem Biophys Res Commun 410, 466-470, https://doi.org/10.1016/j.bbrc.2011.05.161 (2011).

28. Takahashi, A. et al. Creation and biochemical analysis of a broad-specific claudin binder. Biomaterials 33, 3464-3474, https://doi. org/10.1016/j.biomaterials.2012.01.017 (2012).

29. Kojima, T. et al. Claudin-binder C-CPE mutants enhance permeability of insulin across human nasal epithelial cells. Drug Deliv 23, 2703-2710, https://doi.org/10.3109/10717544.2015.1050530 (2016).

30. Moulin, G. et al. A comparison of antimicrobial usage in human and veterinary medicine in France from 1999 to 2005. J Antimicrob Chemother 62, 617-625, https://doi.org/10.1093/jac/dkn213 (2008).

31. Buchner, M. et al. Identification of FOXM1 as a therapeutic target in B-cell lineage acute lymphoblastic leukaemia. Nat Commun 6, 6471, https://doi.org/10.1038/ncomms7471 (2015)

32. Newick, K. et al. Peroxiredoxin 3 is a redox-dependent target of thiostrepton in malignant mesothelioma cells. PLoS One 7, e39404, https://doi.org/10.1371/journal.pone.0039404 (2012).

33. Lok, G. T. et al. Aberrant activation of ERK/FOXM1 signaling cascade triggers the cell migration/invasion in ovarian cancer cells. PLoS One 6, e23790, https://doi.org/10.1371/journal.pone.0023790 (2011).

34. Ju, S. Y., Huang, C. Y., Huang, W. C. \& Su, Y. Identification of thiostrepton as a novel therapeutic agent that targets human colon cancer stem cells. Cell Death Dis 6, e1801, https://doi.org/10.1038/cddis.2015.155 (2015).

35. Nicolaou, K. C. et al. Discovery of a biologically active thiostrepton fragment. J Am Chem Soc 127, 15042-15044, https://doi. org/10.1021/ja0552803 (2005).

36. Bhat, U. G., Zipfel, P. A., Tyler, D. S. \& Gartel, A. L. Novel anticancer compounds induce apoptosis in melanoma cells. Cell Cycle 7, 1851-1855, https://doi.org/10.4161/cc.7.12.6032 (2008).

37. Qiao, S. et al. Thiostrepton is an inducer of oxidative and proteotoxic stress that impairs viability of human melanoma cells but not primary melanocytes. Biochem Pharmacol 83, 1229-1240, https://doi.org/10.1016/j.bcp.2012.01.027 (2012).

38. Kwok, J. M. et al. Thiostrepton selectively targets breast cancer cells through inhibition of forkhead box M1 expression. Mol Cancer Ther 7, 2022-2032, https://doi.org/10.1158/1535-7163.MCT-08-0188 (2008).

39. Hashimoto, Y., Yagi, K. \& Kondoh, M. Roles of the first-generation claudin binder, Clostridium perfringens enterotoxin, in the diagnosis and claudin-targeted treatment of epithelium-derived cancers. Pflugers Arch 469, 45-53, https://doi.org/10.1007/s00424016-1878-6 (2017).

40. Shinoda, T. et al. Structural basis for disruption of claudin assembly in tight junctions by an enterotoxin. Sci Rep 6, 33632, https:// doi.org/10.1038/srep33632 (2016).

41. Suzuki, H., Tani, K. \& Fujiyoshi, Y. Crystal structures of claudins: insights into their intermolecular interactions. Ann N Y Acad Sci. https://doi.org/10.1111/nyas.13371 (2017).

42. Osanai, M., Takasawa, A., Murata, M. \& Sawada, N. Claudins in cancer: bench to bedside. Pflugers Arch 469, 55-67, https://doi. org/10.1007/s00424-016-1877-7 (2017).

43. Tamagawa, H. et al. Characteristics of claudin expression in follicle-associated epithelium of Peyer's patches: preferential localization of claudin-4 at the apex of the dome region. Lab Invest 83, 1045-1053 (2003).

44. Kakutani, H. et al. Mucosal vaccination using claudin-4-targeting. Biomaterials 31, 5463-5471, https://doi.org/10.1016/j. biomaterials.2010.03.047 (2010)

45. Michl, P. et al. Claudin-4: a new target for pancreatic cancer treatment using Clostridium perfringens enterotoxin. Gastroenterology 121, 678-684 (2001).

46. Kojima, T., Kyuno, D. \& Sawada, N. Targeting claudin-4 in human pancreatic cancer. Expert Opin Ther Targets 16, 881-887, https:// doi.org/10.1517/14728222.2012.708340 (2012)

47. Cocco, E. et al. Dual-Targeting Nanoparticles for In Vivo Delivery of Suicide Genes to Chemotherapy-Resistant Ovarian Cancer Cells. Mol Cancer Ther 16, 323-333, https://doi.org/10.1158/1535-7163.MCT-16-0501 (2017).

48. Rajapaksa, T. E., Stover-Hamer, M., Fernandez, X., Eckelhoefer, H. A. \& Lo, D. D. Claudin 4-targeted protein incorporated into PLGA nanoparticles can mediate M cell targeted delivery. J Control Release 142, 196-205, https://doi.org/10.1016/j. jconrel.2009.10.033 (2010).

49. Suzuki, H. et al. C-Terminal Clostridium perfringens Enterotoxin-Mediated Antigen Delivery for Nasal Pneumococcal Vaccine. PLoS One 10, e0126352, https://doi.org/10.1371/journal.pone.0126352 (2015).

50. Watari, A., Hasegawa, M., Yagi, K. \& Kondoh, M. Checkpoint Kinase 1 Activation Enhances Intestinal Epithelial Barrier Function via Regulation of Claudin-5 Expression. PLoS One 11, e0145631, https://doi.org/10.1371/journal.pone.0145631 (2016). 


\section{Acknowledgements}

We thank Yasue Matsunaga and Kazuto Nunomura for their technical assistance in TR-FRET screening and Kaori Ikuma and Shizuka Yamagishi for their technical assistance in animal experiments. This work was supported by JSPS KAKENHI Grant No. 16K08370; by the Yokoyama Rinsho Yakuri Foundation; by the Research Foundation for Pharmaceutical Sciences; by the Takeda Science Foundation; by the Platform for Drug Discovery, Informatics and Structural Life Science of the Ministry of Education, Culture, Sports, Science and Technology, Japan; and by the Basic Science and Platform Technology Program for innovative Biological Medicine from AMED.

\section{Author Contributions}

A.W., K.Y. and M.K. designed the research. K.M., H.S., N.K. and T.T. provided materials. A.W., M.K., Y.S., K.H., Y.Y. and H.T. performed the experiments. A.W., M.K., H.T., K.Y. and M.K. analysed the data; A.W. and M.K. wrote the paper. All authors analysed the results and approved the final version of the manuscript.

\section{Additional Information}

Supplementary information accompanies this paper at https://doi.org/10.1038/s41598-017-15108-y.

Competing Interests: The authors declare that they have no competing interests.

Publisher's note: Springer Nature remains neutral with regard to jurisdictional claims in published maps and institutional affiliations.

(c) (i) Open Access This article is licensed under a Creative Commons Attribution 4.0 International License, which permits use, sharing, adaptation, distribution and reproduction in any medium or format, as long as you give appropriate credit to the original author(s) and the source, provide a link to the Creative Commons license, and indicate if changes were made. The images or other third party material in this article are included in the article's Creative Commons license, unless indicated otherwise in a credit line to the material. If material is not included in the article's Creative Commons license and your intended use is not permitted by statutory regulation or exceeds the permitted use, you will need to obtain permission directly from the copyright holder. To view a copy of this license, visit http://creativecommons.org/licenses/by/4.0/.

(C) The Author(s) 2017 